\title{
Alleviation of carbon catabolite repression in Enterobacter aerogenes for efficient utilization of sugarcane molasses for 2,3-butanediol production
}

Moo-Young Jung ${ }^{1}$, Hwi-Min Jung ${ }^{1}$, Jinwon Lee ${ }^{2}$ and Min-Kyu Oh ${ }^{1 *}$

\begin{abstract}
Background: Due to its cost-effectiveness and rich sugar composition, sugarcane molasses is considered to be a promising carbon source for biorefinery. However, the sugar mixture in sugarcane molasses is not consumed as efficiently as glucose in microbial fermentation due to complex interactions among their utilizing pathways, such as carbon catabolite repression (CCR). In this study, 2,3-butanediol-producing Enterobacter aerogenes was engineered to alleviate CCR and improve sugar utilization by modulating its carbon preference.

Results: The gene encoding catabolite repressor/activator ( $\mathrm{Cra}$ ) was deleted in the genome of E. aerogenes to increase the fructose consumption rate. However, the deletion mutation repressed sucrose utilization, resulting in the accumulation of sucrose in the fermentation medium. Cra regulation on expression of the scrAB operon involved in sucrose catabolism was verified by reverse transcription and real-time PCR, and the efficiency of sucrose utilization was restored by disrupting the scrR gene and overexpressing the $s c r A B$ operon. In addition, overexpression of the pts $G$ gene involved in glucose utilization enhanced the glucose preference among mixed sugars, which relieved glucose accumulation in fed-batch fermentation. In fed-batch fermentation using sugarcane molasses, the maximum titer of 2,3-butanediol production by the mutant reached $140.0 \mathrm{~g} / \mathrm{L}$ at $54 \mathrm{~h}$, which was by far the highest titer of 2,3-butanediol with E. aerogenes achieved through genetic engineering.
\end{abstract}

Conclusions: We have developed genetically engineered E. aerogenes as a 2,3-butanediol producer that efficiently utilizes sugarcane molasses. The fermentation efficiency was dramatically improved by the alleviation of CCR and modulation of carbon preference. These results offer a metabolic engineering approach for achieving highly efficient utilization of mixed sugars for the biorefinery industry.

Keywords: 2,3-Butanediol, Enterobacter aerogenes, Sugarcane molasses, Fed-batch fermentation, Catabolite repressor/activator, Carbon catabolite repression

\section{Background}

The development of biorefineries has attracted a great deal of interest due to increasing energy costs and environmental concerns resulting from fossil fuel utilization $[1,2]$. As an example, microbial production of 2,3-butanediol has been intensively studied in the past few years

\footnotetext{
*Correspondence: mkoh@korea.ac.kr

1 Department of Chemical and Biological Engineering, Korea University, Seoul, Republic of Korea

Full list of author information is available at the end of the article
}

due to its multiple industrial applications, including the production of synthetic rubber, plasticizers, fuel additives, and fumigants $[3,4]$. In microbial fermentation for 2,3-butanediol production, the carbon source is one of the major drivers of cost. Therefore, much effort has been made to find inexpensive feedstocks, such as corncob [5], jatropha hulls [6], Jerusalem artichoke tubers [7], and molasses [8]. In general, most biomass derived from lignocellulose and waste materials contains a few mixed sugars. For example, the hydrolysis of lignocellulosic 
biomass produces a mixture of sugars containing glucose, xylose, and arabinose [9]. The main carbohydrate of Jerusalem artichoke tuber is inulin, which can be hydrolyzed by inulinase to fructose and glucose [10].

When provided with a mixture of different carbon sources, most microorganisms prefer to use one carbon source for the fastest growth. The presence of preferred carbon sources often prevents the utilization of secondary substrates by the regulatory mechanisms, including transcription activation or repression of certain genes concerned with the use of alternative carbon sources, which is called carbon catabolite repression (CCR) [11, 12]. In enteric bacteria, two dominant CCR mechanisms involve transcriptional regulation by cyclic AMP receptor protein (Crp) and by catabolite repressor/activator (Cra) [13]. Crp is known to regulate the genes involved in carbon metabolism, such as lactose, arabinose, mannose, glucosamine, and amino sugars operons, in response to the depletion of preferred carbon source $[14,15]$. On the other hand, Cra generally regulates carbon flux through by repression of genes encoding glycolytic pathway enzymes, or by activating key genes involved in the Krebs cycle, glyoxylate shunt, gluconeogenic pathways and electron transfer [16]. After the preferred carbon source is depleted, an intermediate lag-phase is caused by a shift in the catabolic system to utilize a secondary sugar. Because the catabolic characteristic of microbial fermentation with mixed sugars results in a delayed and complicated fermentation process, alleviation of CCR of a host microorganism is very helpful for improving its fermentation efficiency [17].

Sugarcane molasses contains several mixed sugars, a dominant amount of sucrose, and similar amounts of glucose and fructose. It is considered to be a promising feedstock for biorefinery due to its rich sugar content and cost-effectiveness [18]. In our previous study, disruption of the $s c r R$ gene, a transcriptional repressor of the $s c r$ regulon for sucrose catabolism, was conducted in Enterobacter aerogenes for efficient utilization of sucrose in sugarcane molasses for 2,3-butanediol fermentation [19]. The $s c r R$ mutation increased the sucrose consumption rate significantly, resulting in 2,3-butanediol production from sugarcane molasses that was enhanced by $56.8 \%$ in batch fermentation over its parent strain. In addition, $98.7 \mathrm{~g} / \mathrm{L}$ of 2,3-butanediol production was achieved at $36 \mathrm{~h}$ of fed-batch fermentation with molasses feeding. However, despite these advances, several obstacles remain for more efficient utilization of sugarcane molasses in fed-batch fermentation. First, the efficiency of fructose utilization in the $s c r R$ mutant was relatively reduced, which resulted in a significant amount $(\sim 30 \mathrm{~g} / \mathrm{L})$ of fructose accumulation in the medium after the fermentation period. Second, the fermentation duration of the scrR mutant with sugarcane molasses for 2,3-butanediol production was shorter by $33.3 \%$ than that with glucose, which might be caused by cell stress from the repeated catabolic shift between consumed sugars (glucose and sucrose), according to the feeding of sugarcane molasses.

Therefore, the purpose of this study was to develop a 2,3-butanediol-producing mutant that can utilize all three sugars contained in sugarcane molasses efficiently and simultaneously. As shown in Fig. 1, all sugars in molasses (glucose, fructose, and sucrose) are transported into cells by the phosphotransferase system (PTS) and then converted to intermediates, such as glucose6-phosphate (G6P), fructose-6-phosphate (F6P), and fructose-1,6-bisphosphate (F1,6BP), involved in the preparatory phase of glycolysis [20-23]. Transcription of the fruBKA operon, encoding genes for fructose uptake and utilization, was negatively regulated by Cra, previously

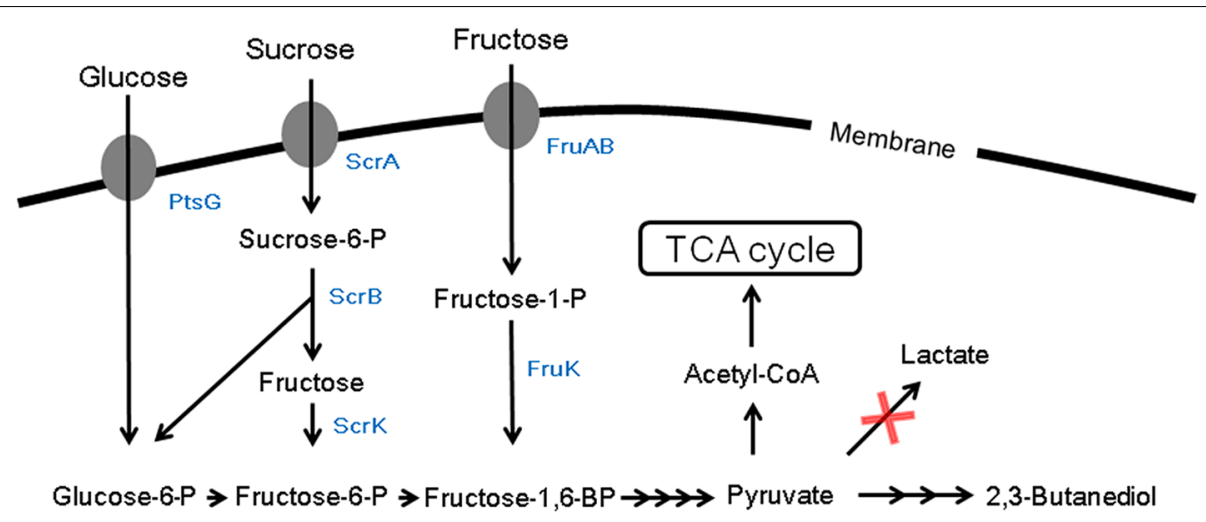

Fig. 1 Catabolic pathway of PTS-mediated sugars contained in sugarcane molasses in E. aerogenes. Symbols represent deleted genes (red cross). ScrA Ell transport protein for sucrose, ScrB sucrose-6-phosphate hydrolase, Scrk fructokinase, PtsG ElIBCGlc complex, FruA EllBCfru complex, FruB EllA fru component, FruK fructose-1-phosphate kinase. 
designated as fruR [24]. When glucose is depleted, Cra activates the metabolic pathways utilizing gluconeogenic carbon sources, such as acetate, pyruvate, glycerol, and glucogenic amino acids $[25,26]$.

In this study, deletion of the cra gene in E. aerogenes showed a much higher fructose utilization rate, but significantly retarded sucrose utilization. The reduced sucrose consumption in cra-deficient $E$. aerogenes was examined by reverse transcription real-time PCR. In addition, the carbon preference and efficiency were modulated by overexpression of the key genes involved in each carbon catabolism, which reduced the accumulation of certain carbon sources in fed-batch fermentation. The catabolic regulation of carbohydrates involved in sugarcane molasses and the strategies for constructing 2,3-butanediol production strain are shown in the Additional file 1.

\section{Results and discussion}

\section{Effects of cra deletion on sugar utilization and metabolite production}

To improve fructose utilization, disruption of $\mathrm{cra}$ was performed. An open-reading frame that contained a 95\% amino acid sequence identity to Cra of Escherichia coli K-12 MG1655 was identified from the E. aerogenes genome using BLAST (Basic Local Alignment Search Tool). The deletion of $\mathrm{cra}$ was performed from EMY-01 $(\triangle l d h A)$ and EMY-68 $(\triangle l d h A \Delta s c r R)$, respectively, and was verified by colony PCR (data not shown). The resulting strains were named EMY-69 $(\Delta l d h A \Delta c r a)$ and
EMY-70 $(\Delta l d h A \Delta s c r R \Delta c r a)$, respectively (Table 1$)$. In our previous study, the $l d h A$-deficient $E$. aerogenes mutant (EMY-01) showed improved carbon flux and NADH availability for 2,3-butanediol production [27]. In addition, acidification of the culture medium was alleviated due to reduced lactate production. Because of these advantages, EMY-01 was used as a control strain in this study.

Flask cultivations of EMY-01, EMY-68, EMY-69, and EMY-70 were conducted with $80 \mathrm{~g} / \mathrm{L}$ of three different carbon sources (fructose, glucose, and sucrose) for $10 \mathrm{~h}$ to confirm the effect of cra deletion on sugar utilization (Fig. 2; Additional file 2). The removal of $s c r R$, a transcriptional repressor of the $s c r$ regulon for sucrose catabolism, caused considerable enhancement of sucrose utilization, but did not affect the utilization of fructose and glucose, in accordance with a previous report [19]. The disruption of $\mathrm{cra}$ did not affect the consumption of glucose, while significantly increasing fructose utilization by 32.9 and $39.0 \%$ in EMY-69 and EMY-70, respectively (Fig. 2a, b). The improvement in fructose utilization increased 2,3-butanediol production by 32.5 and $35.1 \%$, respectively. Interestingly, the removal of cra repressed the utilization of sucrose significantly. The sucrose consumption of EMY-69 and EMY-70 was reduced by 58.2 and $24.6 \%$, respectively, compared to that of their parent strains (Fig. 2c).

The effect of cra mutation was also confirmed in flask cultivation with a mixture of the three sugars. As shown in Fig. 3a, b, glucose was the preferred carbon source

Table 1 Strains and plasmids used in this study

\begin{tabular}{|c|c|c|}
\hline Strains or plasmids & Genotype or relevant characteristics & Source or reference \\
\hline \multicolumn{3}{|l|}{ Strain } \\
\hline E. coli DH5 $\alpha$ & & Invitrogen \\
\hline $\begin{array}{l}\text { E. aerogenes } \\
\text { KCTC } 2190\end{array}$ & Wild type & Korean Collection for Type Culture \\
\hline EMY-01 & E. aerogenes KCTC $2190 \triangle / d h A$ & [27] \\
\hline EMY-68 & E. aerogenes KCTC $2190 \triangle /$ dhA $\triangle \mathrm{scr} R$ & {$[19]$} \\
\hline EMY-69 & E. aerogenes KCTC $2190 \Delta / d h A \Delta c r a$ & This study \\
\hline EMY-70 & E. aerogenes KCTC $2190 \Delta / d h A \Delta s c r R \Delta c r a$ & This study \\
\hline EMY-70S & Plasmid-based scrAB overexpression strain of EMY-70 by introduction of pZS21MCS::scrAB & This study \\
\hline EMY-70SP & Plasmid-based pts G overexpression strain of EMY-70S by introduction of pZA31MCS::ptsG & This study \\
\hline \multicolumn{3}{|l|}{ Plasmid } \\
\hline pKM208 & lacl, $\lambda$ Red + Gam-producing vector, tac_promoter, f1_ori, Amp $p^{R}$ & Addgene \\
\hline pCP20 & FLP recombinase-producing vector, c1857, pSC101 ori, $\mathrm{Amp}^{\mathrm{R}}, \mathrm{Cm}^{\mathrm{R}}$ & {$[40]$} \\
\hline pKD4 & FRT flanked resistance cassette involved vector, oriR $\gamma, \mathrm{Km}^{\mathrm{R}}$ & {$[40]$} \\
\hline pZA31MCS & E. coli-K. pneumoniae shuttle vector, $\mathrm{P}_{\text {Lteto-1 }}, \mathrm{p} 15 \mathrm{~A}$ ori, $\mathrm{Cm}^{\mathrm{R}}$ & Expressys \\
\hline pZS21MCS & E. coli-K. pneumoniae shuttle vector, $\mathrm{P}_{\text {Lteto-1, }}$ pSC101 ori, $\mathrm{Km}^{\mathrm{R}}$ & Expressys \\
\hline pZA31MCS::ptsG & pZA31MCS derivative containing pts $G, P_{\text {Lteto-1, }}$ p15A ori, $\mathrm{Cm}^{\mathrm{R}}$ & This study \\
\hline pZS21MCS::sCrAB & pZS21MCS derivative containing SCrAB, $\mathrm{P}_{\text {Lteto-1, }}$ pSC101 ori, $\mathrm{Km}^{\mathrm{R}}$ & This study \\
\hline
\end{tabular}





Fig. 2 Comparison of consumed concentration of $\mathbf{a}$ glucose, $\mathbf{b}$ fructose, and $\mathbf{c}$ sucrose by E. aerogenes mutants in 10 h of flask cultivation. Gray bars concentration of consumed sugars; black bars 2,3-butanediol production. Error bars represent the standard deviations of three experiments. 



Fig. 3 Comparison of flask cultivations of a EMY-01, b EMY-68, c EMY-69, d EMY-70, e EMY-70S, and $\mathbf{f}$ EMY-70SP with the consumption of sugars. Error bars represent the standard deviations of three experiments.

of EMY-01 and EMY-68. After glucose was depleted, EMY-01 utilized fructose and sucrose at similar rates, while EMY-68 consumed sucrose faster than fructose due to the disruption of $s c r R$. Meanwhile, the most preferred sugar of the cra-deficient mutants was fructose.
The fructose consumption rates of EMY-69 and EMY-70 increased by 124.0 and $420.5 \%$, respectively, compared to that of their parent strains in $10 \mathrm{~h}$ of flask cultivation with mixed sugars. Furthermore, a significant decrease (81.3\%) in the sucrose consumption of EMY-69 was also 
observed with $12 \mathrm{~h}$ of flask cultivation with mixed carbon sources, resulting in a $24.1 \%$ reduction of 2,3-butanediol production (Fig. 3; Additional file 3).

Transcription of the fructose operon is repressed when Cra is bound to the operator, which is located on the downstream region of the RNA polymerase binding site [24]. Whereas, derepression of the fructose operon takes place when cytoplasmic glycolytic catabolites, such as fructose-1-phosphate and fructose-1,6-bisphosphate, bind to the Cra protein, causing it to dissociate from the operator. Our results demonstrate that cra deletion improves the consumption rate of fructose significantly and removes CCR with respect to fructose utilization, which increased 2,3-butanediol production. At the same time, a significant decrease in the sucrose uptake rate was observed with deletion of $\mathrm{Cra}$, indicating a significant interaction between the scr regulon and Cra. The efficient utilization of sucrose is essential for maximizing the use of sugarcane molasses, because $70 \%$ of the carbon contained in sugarcane molasses is sucrose. However, the regulation of sucrose catabolism by Cra remains unexplored in enteric bacteria.

\section{Regulation of genes involved in sucrose catabolism in cra-deficient $E$. aerogenes}

To observe the mechanism of Cra regulation of sucrose catabolism, reverse transcription and real-time PCR was conducted with a cra-deficient mutant, which was grown in sucrose medium. Six genes related to sucrose utilization ( $s c r A, s c r B, s c r R$, scrK, $c r a$, and $c r p$ ) were selected for the reverse transcription and real-time PCR assay. The transcription level of genes involved in the sucrose catabolism of EMY-01 and EMY-69 was compared in the midexponential growth phase $(6 \mathrm{~h})$, using gap $A$ as a reference gene [28]. As shown in Fig. 4, there were no significant differences in the relative expression levels of $s c r R, s c r K$, or crp in the strains, whereas the transcription levels of $s c r A$ and $s c r B$ decreased by 32.0 and $24.9 \%$, respectively, by disruption of the cra gene.

In E. aerogenes, the scr regulon for sucrose catabolism consists of four genes, which encode ATP-dependent fructokinase (ScrK), PTS EII transport protein (ScrA), sucrose-6-phosphate hydrolase (ScrB), and sucrosedependent regulator (ScrR) [29, 30]. The scr regulon contains two independent promoters, one in front of the $s c r K$ and a second in front of the $s c r A B$ operon, both of which are negatively regulated by ScrR (Additional file 1) [31]. In E. coli, the sequence of the Cra-binding site (Cra box), TGAAACGTTTCA, has been well characterized [16]. Interestingly, a sequence similar to the consensus sequence of the Cra box, TAAAACGTTTTA, was found in the $\operatorname{scr} A B$ promoter region of $E$. aerogenes. In addition,

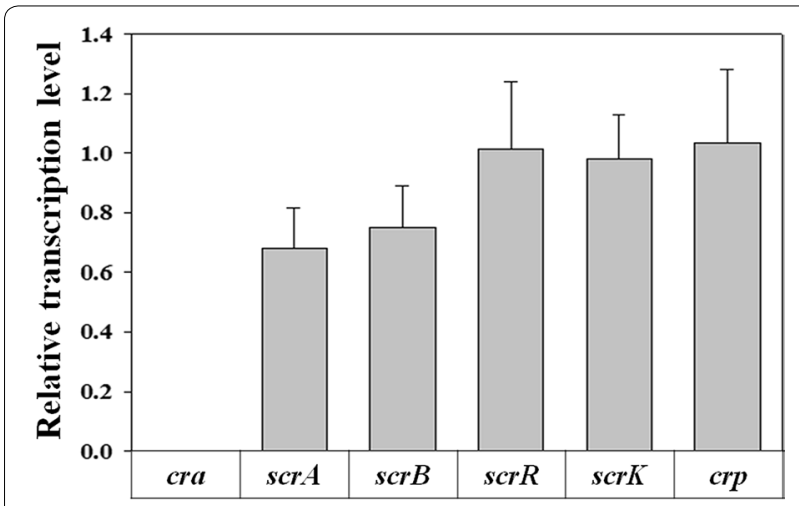

Fig. 4 Real-time PCR results of genes involved in sucrose catabolism by the deletion of the cra gene. The transcription level of genes in EMY01 was used as the control. Error bars represent the standard deviations of three experiments.

no significant change in the transcription level of crp was observed in our results when cra was disrupted. These results demonstrate that Cra directly binds to the $\operatorname{scr} A B$ promoter region to regulate its expression.

The CCR mechanisms by Crp and Cra are complex and interconnected. Indeed, Cra regulates the utilization of several carbon sources, such as xylose, glycerol, lactose, and sorbitol, through activating expression of the crp gene, even though Cra does not regulate the transcription of relevant catabolic genes directly [13]. In this experiment, we demonstrated the sugar consumption genes can be modulated by cra deletion, and that regulation is independent with Crp. This result is another example of controlling CCR. Previously Ji et al. tried it by overexpression of crp(in) gene, which made Klebsiella oxytoca utilize glucose and xylose simultaneously and resulted in higher growth and 2,3-butanediol productivity [32].

\section{Effects of $s c r A B$ and ptsG overexpression on the sugar utilization preference in sugarcane molasses}

The decreased sucrose utilization by deletion of cra in EMY-69 was partially relieved by $s c r R$ mutation, shown in EMY-70 (Fig. 2c). However, sucrose utilization in that strain was not as efficient as glucose or fructose. Therefore, the $\operatorname{scr} A B$ operon was cloned in the expression vector $\mathrm{pZS} 21 \mathrm{MCS}$ and overexpressed in EMY-70 to increase sucrose utilization. The resulting strain, EMY$70 S$, showed considerably enhanced sucrose utilization, as shown in Fig. 2c. EMY-70S consumed $61.5 \mathrm{~g} / \mathrm{L}$ sucrose for $10 \mathrm{~h}$ of flask culture using sucrose as a sole carbon source, representing an increase of $42.2 \%$ compared to that of EMY-70. In flask cultivation of EMY-70S with mixed sugars, the consumption rate of sucrose improved to a similar rate as that of fructose consumption (Fig. 3e). 
Interestingly, glucose was the carbon source with the lowest priority among the three carbon sources in EMY70S. The consumption of glucose was reduced by $46.0 \%$ compared to that of its parent strain (Figs. 3d, e).

Thus, we attempted to resolve the reduced efficiency of glucose utilization in EMY-70S at the genetic level. In glucose catabolism, the EIIBC ${ }^{\text {Glc }}$ complex encoded by ptsG is known to be a major controller of glucose flux in $E$. coli $[33,34]$. Therefore, a strain overexpressing the ptsG gene was constructed from EMY-70S and named EMY-70SP. Although the overexpression of ptsG did not affect the carbon source utilization of a sole carbohydrate, such as fructose, glucose, or sucrose, the efficiency of glucose utilization was restored in flask cultivations with a mixture of sugars (Figs. 2, 3f). In EMY-70SP, the consumption rate of sucrose was slightly reduced. Among the carbohydrate consumption pathways (Fig. 1), G6P is converted not only from glucose by the pts $G$ gene product, but also from sucrose by a series of enzymes involved in the $\operatorname{scr} A B$ operon. Therefore, the result suggested that overexpression of the $p t s G$ gene presumably activated glucose transport with G6P formation, which resulted in the relative repression of G6P synthesis derived from sucrose.

As shown in the Additional file 3, there were no significant differences in the total concentration of the carbon source consumed by E. aerogenes mutants when the flask cultivations were performed with mixed sugars. These results indicate that the maximum carbon source consumption through glycolysis might be reached, but the sugar preference was modulated by genetic engineering. Still, predictable control of sugar preference would be very beneficial for efficient utilization of biomass that contains a variety of carbon sources. This is because the concentration and content ratio of sugars contained in biomass vary depending on the type of biomass resources and local climate and soil conditions even the same type of biomass [35-37].

\section{Fed-batch fermentation with $E$. aerogenes mutants using sugarcane molasses}

To improve 2,3-butanediol production from sugarcane molasses, a fed-batch fermentation was performed with EMY-70S. As shown in Fig. 5c and Additional file 4, 2,3-butanediol production reached $140.0 \mathrm{~g} / \mathrm{L}$ at $54 \mathrm{~h}$ of cultivation. To the best of our knowledge, this was by far the highest titer of 2,3-butanediol with E. aerogenes achieved by genetic engineering, and was $18.6 \%$ higher than that with glucose as a carbon source [27]. A total of $361.7 \mathrm{~g} / \mathrm{L}$ of sugars in sugarcane molasses was consumed. Among the major byproducts, ethanol production reached $13.7 \mathrm{~g} / \mathrm{L}$ at $14 \mathrm{~h}$ of fed-batch fermentation, after which its concentration was gradually decreased to $1.6 \mathrm{~g} / \mathrm{L}$ at $54 \mathrm{~h}$, in accordance with a previous report [19]. The production of acetoin, which is a precursor of 2,3-butanediol, was under $2.0 \mathrm{~g} / \mathrm{L}$ until $40 \mathrm{~h}$ of fermentation, but its titer steadily increased to $8.6 \mathrm{~g} / \mathrm{L}$ at $54 \mathrm{~h}$. The acetoin accumulation is due to the reduced activity of 2,3-butanediol dehydrogenase (BudC) by a high concentration of 2,3-butanediol in the fermentation broth. The production of 2,3-butanediol by EMY$70 \mathrm{~S}$ was prolonged until $54 \mathrm{~h}$ of cultivation, which was $50 \%$ longer than the fed-batch with both EMY-01 and EMY-68 (Figs. 5, 6). Even at $36 \mathrm{~h}$ of fed-batch fermentation, 2,3-butanediol productivity $(\mathrm{g} / \mathrm{L} / \mathrm{h})$ of EMY-70S increased by $23.8 \%$ compared to that of EMY-68. These results indicate that disruption of both $s c r R$ and $c r a$ genes presumably relieved the cell stress due to repeated catabolic shift according to the feeding of sugarcane molasses in fed-batch fermentation. However, there was significant accumulation $(30 \mathrm{~g} / \mathrm{L})$ of glucose in the medium after the fermentation period (Fig. 6c).

To observe the effect of $p t s G$ overexpression on glucose consumption, fed-batch fermentation was performed with EMY-70SP. As shown in Fig. 5d and Additional file $4,335.58 \mathrm{~g} / \mathrm{L}$ of sugars in sugarcane molasses was consumed, and 2,3-butanediol production reached $129.36 \mathrm{~g} / \mathrm{L}$ at $54 \mathrm{~h}$ of cultivation. Although 2,3-butanediol production was decreased by $7.6 \%$ compared to that of EMY-70S, all three carbons in sugarcane molasses were used efficiently (Fig. 6d). The metabolic burden according to overexpression of the $\operatorname{scr} A B$ operon and $p t s G$ gene may be the main reason for the slight reduction observed in fed-batch fermentation with EMY-70SP. These results demonstrate the advantage of genetic engineering in utilizing mixtures of sugars contained in biomass under the fermentation process [38].

\section{Conclusions}

The efficient utilization of biomass is necessary in order to develop an economic biorefinery industry. In this study, sugarcane molasses was used as a cheap feedstock for 2,3-butanediol production with metabolically engineered E. aerogenes. For efficient utilization of sugarcane molasses, the removal of transcriptional repressors enabled the mutant strain to metabolize all sugars in sugarcane molasses simultaneously, which increased fermentation duration and 2,3-butanediol productivity. In addition, the carbon preference was modulated by overexpression of key genes involved in each carbon catabolism, which relieved the accumulation of certain carbon sources in fed-batch fermentation. The metabolic engineering approach provided much higher 2,3-butanediol production and efficient utilization of carbon sources involved in sugarcane molasses. The strategy developed 


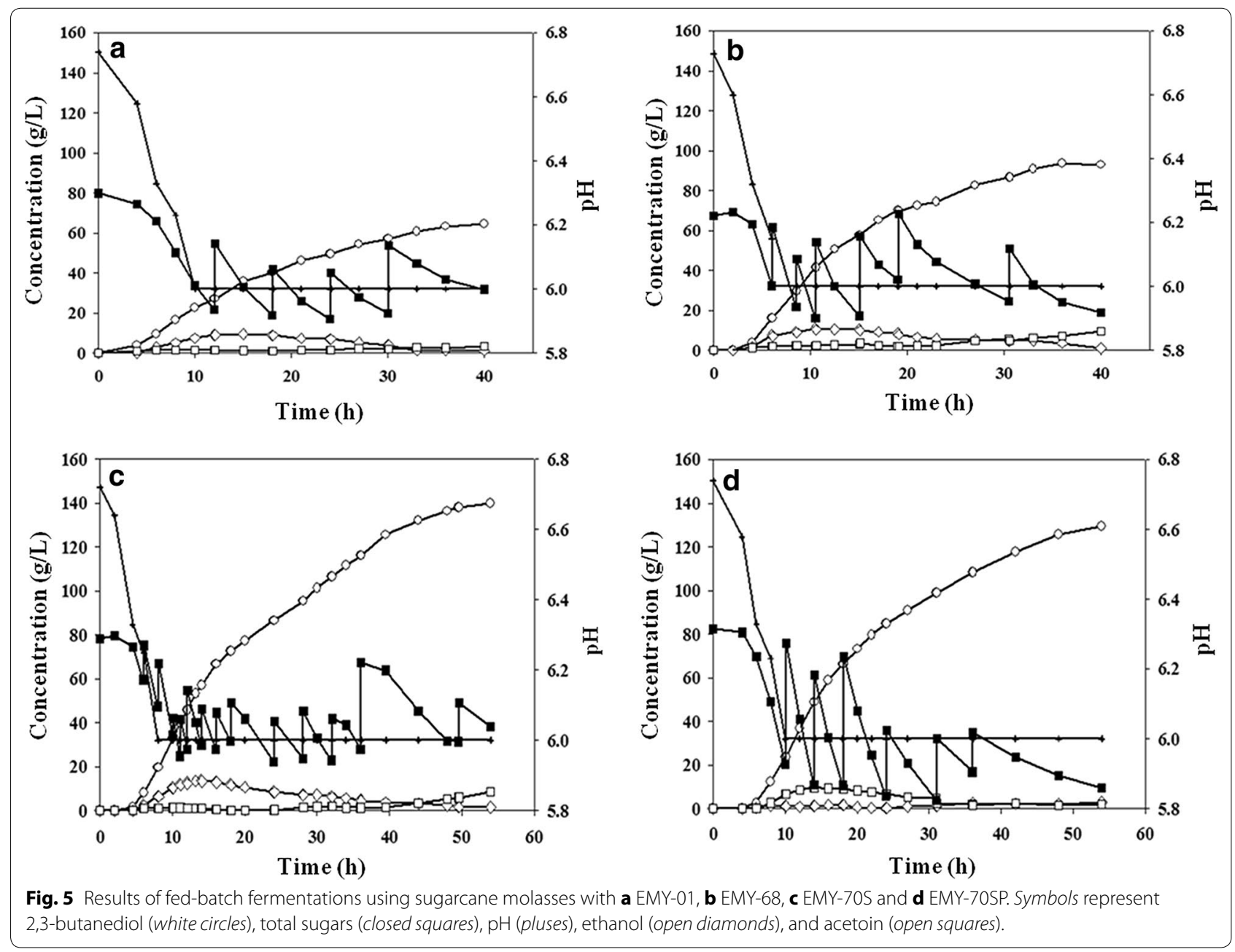

in this study is a promising approach for the biorefinery industry, through employing sugar mixtures derived from inexpensive biomass.

\section{Methods}

\section{Construction of the gene-deficient mutants}

All $E$. aerogenes strains were derived from the wild-type strain KCTC 2190 (Korean Collection for Type Culture), for which the genome has been sequenced [39]. Previously, the lactate dehydrogenase (LdhA, accession number YP_004594301.1) and sucrose regulator (ScrR, accession number YP_004593287.1) were disrupted from the KCTC 2190 genome by using a $\lambda$ red recombination method [40], generating EMY-01 $(\triangle l d h A)$ [27] and EMY-68 ( $\triangle l d h A \Delta s c r R)$ [19], respectively. In this study, the gene encoding catabolite repressor/activator, Cra (previously designated fruR, accession number YP_004592435.1), was deleted from the genome of EMY01 and EMY-68 in a similar manner, and the resulting strains were named EMY-69 $(\Delta l d h A \Delta c r a)$ and EMY-70 $(\triangle l d h A \quad \triangle s c r R \quad \Delta c r a)$, respectively. The cra_FKF_fw and $c r a \_F K F \_r v$ primers were used for cra disruption, which was confirmed by colony PCR with the primers cra_con_A and cra_con_B. All resulting strains, along with primers and plasmids, used in this study are listed in Tables 1 and 2.

\section{Construction of plasmids}

Enterobacter aerogenes KCTC 2190 was cultured in Luria-Bertani medium overnight, and then total genomic DNA was extracted using the Wizard Genomic DNA Purification Kit (Promega, WI, USA). For $s c r A B$ overexpression, the $s c r A B$ fragment (a 2,771-bp segment of truncated $s c r A B$ gene, accession number YP_004593289.1 and YP_004593288.1) was amplified by PCR using the genomic DNA as a template and the primers pZS21 $s c r A B \_f w$ and _rv at an annealing temperature (Tm) of $62.4^{\circ} \mathrm{C}$. The PCR mixture consisted of $100 \mathrm{ng}$ of genomic DNA, $200 \mu \mathrm{mol}$ of dNTPs, 0.5 pmol each primer, $10 \mu \mathrm{L}$ of $5 \times$ Phusion GC buffer, $1.5 \mu \mathrm{L}$ of DMSO, and 1.0 unit 

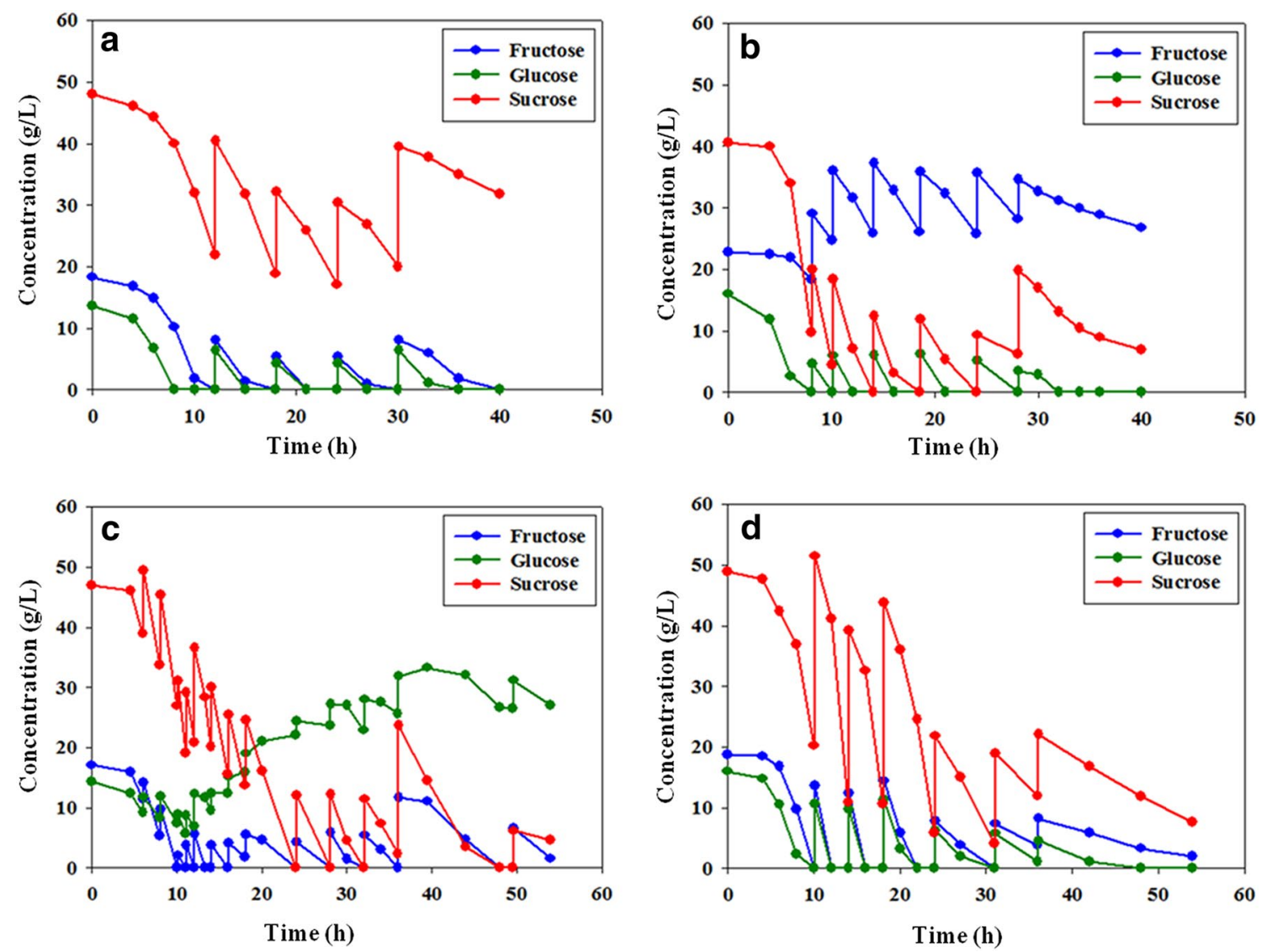

Fig. 6 Comparison of fed-batch fermentations of a EMY-01, b EMY-68, c EMY-70S and $\mathbf{d}$ EMY-70SP with the consumption of sugars.

of Phusion DNA polymerase (NEB, MA, USA) in a total volume of $50 \mu \mathrm{L}$. The amplified DNA fragments were cloned into the pZS21MCS plasmid joined by the Hin$d \mathrm{III}$ and $X m a \mathrm{I}$ restriction sites using the DNA Ligation Kit Mighty Mix (TaKaRa Bio Inc., Shiga, Japan). The $E$. coli strain DH5 $\alpha$ was used for amplification and confirmation of the constructed plasmid. The resulting plasmid was verified by sequencing. The $p t s G$ fragment (a 1,434bp segment of truncated $p t s G$ gene, accession number YP_004593473.1) was amplified by PCR using the corresponding primers. The PCR mixture composition and procedure were the same as that of $\operatorname{scr} A B$, but the annealing temperature was $64^{\circ} \mathrm{C}$. The amplified $p t s G$ fragments were cloned into the pZA31MCS plasmid after digestion with HindIII and BamHI. The plasmids and primers used in this study are listed in Tables 1 and 2.

\section{Media and cultivation conditions}

The fermentation medium to produce 2,3-butanediol contained (per L): $3 \mathrm{~g} \mathrm{KH}_{2} \mathrm{PO}_{4}, 6.8 \mathrm{~g} \mathrm{Na}_{2} \mathrm{HPO}_{4}$, $0.75 \mathrm{~g} \mathrm{KCl}, 5.35 \mathrm{~g}\left(\mathrm{NH}_{4}\right)_{2} \mathrm{SO}_{4}, 0.28 \mathrm{~g} \mathrm{Na}_{2} \mathrm{SO}_{4}, 0.26 \mathrm{~g}$ $\mathrm{MgSO}_{4} \cdot 7 \mathrm{H}_{2} \mathrm{O}, 0.42 \mathrm{~g}$ citric acid, $5 \mathrm{~g}$ yeast extract, $10 \mathrm{~g}$ casamino acid, and $0.3 \mathrm{~mL}$ microelement solution (1 L) containing $34.2 \mathrm{~g} \mathrm{ZnCl}_{2}, 2.7 \mathrm{~g} \mathrm{FeCl}_{3} \cdot 6 \mathrm{H}_{2} \mathrm{O}, 10 \mathrm{~g}$ $\mathrm{MnCl}_{2} \cdot 4 \mathrm{H}_{2} \mathrm{O}, 0.85 \mathrm{~g} \mathrm{CuCl}_{2} \cdot 2 \mathrm{H}_{2} \mathrm{O}$, and $0.31 \mathrm{~g} \mathrm{H}_{3} \mathrm{BO}_{3}$, as described previously [19]. In a flask culture, $80 \mathrm{~g} / \mathrm{L}$ of the individual sugars (glucose, fructose, or sucrose) was added to the medium as the sole carbon source, and the total $90 \mathrm{~g} / \mathrm{L}$ mixture of sugars, containing $30 \mathrm{~g} / \mathrm{L}$ each of glucose, fructose, and sucrose, was added to the medium for the sugar mixture cultivation. For $\mathrm{pH}$ neutralization of the flask cultivation, $5 \%$ calcium carbonate $\left(\mathrm{CaCO}_{3}\right)$ was added to the medium before cultivation. The flask, sealed with a silicon stopper, was incubated at $250 \mathrm{rpm}$ and $37^{\circ} \mathrm{C}$ in a $250-\mathrm{mL}$ flask containing $50 \mathrm{~mL}$ medium with appropriate antibiotics or inducers, when needed, at the following concentrations: kanamycin $(50 \mu \mathrm{g} / \mathrm{mL})$, chloramphenicol $(50 \mu \mathrm{g} / \mathrm{mL})$, and anhydrotetracycline $(50 \mathrm{ng} / \mathrm{mL}$ ). The fed-batch fermentation was carried out in a 5-L stirring bioreactor (Bio Control and System, Daejeon, Korea) with a working volume of $3 \mathrm{~L}$. The seed culture prepared previously was inoculated $(5 \%, \mathrm{v} / \mathrm{v})$ into the fermentation medium with an initial $\mathrm{pH}$ of 6.8. The $\mathrm{pH}$ value of the fermentation medium was decreased 
Table 2 Primers used in this study

\begin{tabular}{|c|c|}
\hline Primer name & Primer sequence $\left(5^{\prime} \rightarrow 3^{\prime}\right)^{a}$ \\
\hline cra_FKF_fw & GTATGTCTATTTAATGGTTGTTTTTTGTACTTCTTACCCAAGGGGCAATTGTGTAGGCTGGAGCTGCTTC \\
\hline cra_FKF_rv & CCATCTGGCGAATAACCTACGAAGAATCTTAACCTTTTTTCGCAAATGAACCTCCTTAGTTCCTATTCC \\
\hline cra_con_A & ACGTAAAAACAGCCCGACAC \\
\hline cra_con_B & CGCTTTTTCTTGCACCATTT \\
\hline pZS21_scrAB_fw & TTTAAGCTTATGGATTTTCAACAGATTTCTCG \\
\hline pZS21_scrAB_rv & AAACCCGGGGCCTGAAAGCAAAACGCTTA \\
\hline pZA31_ptsG_fw & TTTAAGCTTACTCAGGAGCACTCTCAATTATGTT \\
\hline pZA31_ptsG_rv & AAAGGATCCTTAGCTATTGCGGATGTACTCA \\
\hline RT_scrA_fw & TCGGCGGTAACCCTTATCTT \\
\hline RT_scrA_rv & ATTAGCCATCGCCCAGATAG \\
\hline RT_scrB_fw & TGCTACACCGGTAATGTGAAAT \\
\hline RT_scrB_rv & TAGAATTCGAAGCCGCTATCC \\
\hline $\mathrm{RT} \_s c r R \_f \mathrm{w}$ & CCGGCGTACAGCTGCTTAT \\
\hline RT_scrR_rv & ACGTAGTACCCCTTCCAGCAA \\
\hline RT_scrk_fw & TCAGCCATCTTTCTTTAGATCC \\
\hline RT_scrk_rv & CGGGAAGTGAATATGCTGTTG \\
\hline RT_cra_fw & CCGTATTGCGAACTATCTGGA \\
\hline RT_cra_rv & TAAACAGCAGTTGCGGCATT \\
\hline RT_crp_fw & ATCAAAGAGCACGCTGATTC \\
\hline RT_crp_rv & CCAGCATCTTCAGAATACGG \\
\hline RT_gapA_fw & TTGGTGTTGACGTTGTTGCT \\
\hline RT_gapA_rv & TTCGTAGGACGCTGCTTTTT \\
\hline
\end{tabular}

${ }^{a}$ Underlined sequences are the homologous sequences with the target genes of E. aerogenes. Restriction sites highlighted in bold.

gradually to 6.0, and then maintained by the automatic addition of $5 \mathrm{M} \mathrm{NaOH}$. During the fed-batch fermentation process, the operating temperature, airflow, and agitation speed were maintained at $37^{\circ} \mathrm{C}, 1.5 \mathrm{vvm}$, and $280 \mathrm{rpm}$, respectively. Antifoam 204 (Sigma, MO, USA) was added when needed. Brazilian sugarcane molasses was used, and its sugar content was $87 \mathrm{~g} / \mathrm{L}$ fructose, $81 \mathrm{~g} / \mathrm{L}$ glucose, and $387 \mathrm{~g} / \mathrm{L}$ sucrose, as described previously [19]. The sterilized sugarcane molasses was fed before the sugar was depleted, and the sugar concentration was maintained under $80 \mathrm{~g} / \mathrm{L}$ during fed-batch fermentation.

\section{Quantification of the transcription level of genes involved in sucrose catabolism by real-time PCR}

Total RNA isolation was performed as previously described [41]. Briefly, cells $\left(1 \times 10^{9}\right)$ were harvested at the mid-exponential phase $(6 \mathrm{~h})$ and, then, the total RNA in the cell was stabilized using the bacterial reagent RNAprotect (Qiagen, Hilden, Germany). Cell lysis was conducted with TE buffer containing lysozyme (400 $\mu \mathrm{g} / \mathrm{mL}$ ), and total RNA isolation was carried out using an RNeasy Mini Kit (Qiagen) following the manufacturer's protocol. To synthesize the cDNA from total RNA, SuperScript ${ }^{\circledR}$ Reverse Transcriptase (Invitrogen,
CA, USA) was used. Real-time PCR was performed using the synthesized cDNA as a template (Model: MJ Mini thermocycler, Software: Opticon Monitor 3, BioRad, CA, USA) with six sets of PCR primers and a SYBR Green mix (Prime Q-Master mix, Genet Bio, Daejon, Korea). The primers used in real-time PCR are listed in Table 2. The transcription levels of the five genes involved in sucrose catabolism were normalized to the housekeeping gene glyceraldehyde 3-phosphate dehydrogenase $(\operatorname{gap} A)$. The experiment was repeated three times independently.

\section{Analysis methods}

Cell density was monitored by measurement of the optical density at $600 \mathrm{~nm}\left(\mathrm{OD}_{600}\right)$ with a UV/Visible spectrophotometer (DU730, Beckman Coulter, CA, USA). The concentrations of metabolites, obtained from cultivation, were measured by high-performance liquid chromatography (Waters HPLC 1500 series, MA, USA), equipped with a refractive index $(\mathrm{RI})$ detector at $45^{\circ} \mathrm{C}$. The amounts of fructose, glucose, and sucrose were measured using a High Performance Carbohydrate Column (Waters) at $35^{\circ} \mathrm{C}$, and $80 \%$ acetonitrile was used as the mobile phase. Organic acids, 2,3-butanediol, acetoin, and ethanol, were measured using a Sugar SH1011 column (Shodex, Tokyo, 
Japan) at $75^{\circ} \mathrm{C}$, and $10 \mathrm{mM}$ sulfuric acid was used as the mobile phase. The flow rate of both mobile phases was maintained at $0.5 \mathrm{~mL} / \mathrm{min}$.

\section{Additional files}

Additional file 1: Figure S1. Regulation mechanism related to the utilization of sugars involved in sugarcane molasses and the strategies for constructing the 2,3-butanediol-producing strain in this study. Symbols represent deleted genes (red cross) and overexpressed genes (blue box).

Additional file 2: Table S1. Comparison of metabolite profiles obtained from E. aerogenes mutants using sole carbon sources in $10 \mathrm{~h}$ of flask cultivation.

Additional file 3: Table S2. Comparison of metabolite profiles obtained from E. aerogenes mutants using mixed carbon sources in $12 \mathrm{~h}$ of flask cultivation.

Additional file 4: Table S3. Comparison of fed-batch fermentation with EMY-01, EMY-68, EMY-70S, and EMY-70SP using sugarcane molasses.

\section{Abbreviations}

Cra: catabolite repressor/activator; CCR: carbon catabolite repression; PTS: phosphotransferase system; G6P: glucose-6-phosphate; F6P: fructose-6-phosphate; F1,6BP: fructose-1,6-bisphosphate; PCR: polymerase chain reaction; Ldh: lactate dehydrogenase; OD: optical density; ScrA: Ell transport protein for sucrose; ScrB: sucrose-6-phosphate hydrolase; Scrk: fructokinase; ScrR: transcriptional repressor of scr regulon; PtsG: ElIBC ${ }^{G l c}$ complex; FruA: EllBC fru complex; FruB: Ell $A^{\text {fru }}$ component; FruK: fructose-1-phosphate kinase; Crp: cyclic AMP receptor protein.

\section{Authors' contributions}

MY Jung designed the study, performed the experiments, analyzed the data, and drafted the manuscript. HM Jung executed the experimental work. JW Lee provided some materials and reagents and helped design the experiments. MK Oh designed the experiments, revised the manuscript, and supervised the project. All authors have read and approved the final manuscript.

\section{Author details}

1 Department of Chemical and Biological Engineering, Korea University, Seoul, Republic of Korea. ${ }^{2}$ Department of Chemical and Biomolecular Engineering, Sogang University, Seoul, Republic of Korea.

\section{Acknowledgements}

This study was supported by the R\&D program of MKE/KEIT (No. 10035578, Development of 2,3-butanediol and derivate production technology for C-Zero bio-platform industry) and the New \& Renewable Energy Core Technology Program of the Korea Institute of Energy Technology Evaluation and Planning (KETEP), granted financial resource from the Ministry of Trade, Industry \& Energy, Republic of Korea. (No. 20143030091040).

\section{Compliance with ethical guidelines}

\section{Competing interests}

The authors declare that they have no competing interests.

Received: 17 February 2015 Accepted: 22 July 2015

Published online: 31 July 2015

\section{References}

1. Ragauskas AJ, Williams CK, Davison BH, Britovsek G, Cairney J, Eckert CA et al (2006) The path forward for biofuels and biomaterials. Science 311:484-489

2. van Haveren J, Scott EL, Sanders J (2008) Bulk chemicals from biomass. Biofuel Bioprod Biorefin 2:41-57
3. Ji XJ, Huang H, Ouyang PK (2011) Microbial 2,3-butanediol production: a state-of-the-art review. Biotechnol Adv 29:351-364

4. Celinska E, Grajek W (2009) Biotechnological production of 2,3-butanediol_current state and prospects. Biotechnol Adv 27:715-725

5. Wang AL, Wang Y, Jiang TY, Li LX, Ma CQ, Xu P (2010) Production of 2,3-butanediol from corncob molasses, a waste by-product in xylitol production. Appl Microbiol Biotechnol 87:965-970

6. Jiang LQ, Fang Z, Guo F, Yang LB (2012) Production of 2,3-butanediol from acid hydrolysates of Jatropha hulls with Klebsiella oxytoca. Bioresour Technol 107:405-410

7. Sun LH, Wang XD, Dai JY, Xiu ZL (2009) Microbial production of 2,3-butanediol from Jerusalem artichoke tubers by Klebsiella pneumoniae. Appl Microbiol Biotechnol 82:847-852

8. Dai JY, Zhao P, Cheng XL, Xiu ZL (2015) Enhanced production of 2,3-butanediol from sugarcane molasses. Appl Biochem Biotechnol 175:3014-3024

9. Mosier N, Wyman C, Dale B, Elander R, Lee YY, Holtzapple M et al (2005) Features of promising technologies for pretreatment of lignocellulosic biomass. Bioresour Technol 96:673-686

10. Gao L, Chi Z, Sheng J, Ni X, Wang L (2007) Single-cell protein production from Jerusalem artichoke extract by a recently isolated marine yeast Cryptococcus aureus G7a and its nutritive analysis. Appl Microbiol Biotechnol 77:825-832

11. Goerke B, Stulke J (2008) Carbon catabolite repression in bacteria: many ways to make the most out of nutrients. Nat Rev Microbiol 6:613-624

12. Deutscher J (2008) The mechanisms of carbon catabolite repression in bacteria. Curr Opin Microbiol 11:87-93

13. Zhang ZG, Aboulwafa M, Saier MH (2014) Regulation of crp gene expression by the catabolite repressor/activator, Cra, in Escherichia coli. J Mol Microbiol Biotechnol 24:135-141

14. Plumbridge J (1998) Control of the expression of the manXYZ operon in Escherichia coli: Mlc is a negative regulator of the mannose PTS. Mol Microbiol 27:369-380

15. Newman JR, Fuqua C (1999) Broad-host-range expression vectors that carry the L-arabinose-inducible Escherichia coli araBAD promoter and the araC regulator. Gene 227:197-203

16. Shimada T, Yamamoto K, Ishihama A (2011) Novel members of the Cra regulon involved in carbon metabolism in Escherichia coli. J Bacteriol 193:649-659

17. Gawand P, Hyland P, Ekins A, Martin VJJ, Mahadevan R (2013) Novel approach to engineer strains for simultaneous sugar utilization. Metab Eng 20:63-72

18. Akaraonye E, Moreno C, Knowles JC, Keshavarz T, Roy I (2012) Poly(3hydroxybutyrate) production by Bacillus cereus SPV using sugarcane molasses as the main carbon source. Biotechnol J 7:293-303

19. Jung MY, Park BS, Lee J, Oh MK (2013) Engineered Enterobacter aerogenes for efficient utilization of sugarcane molasses in 2,3-butanediol production. Bioresour Technol 139:21-27

20. Sprenger GA, Lengeler JW (1988) Analysis of sucrose catabolism in Klebsiella-pneumoniae and in Scr+ derivatives of Escherichia coli K12.J Gen Microbiol 134:1635-1644

21. Kornberg HL (2001) Routes for fructose utilization by Escherichia coli. J Mol Microbiol Biotechechnol 3:355-359

22. Deutscher J, Francke C, Postma PW (2006) How phosphotransferase system-related protein phosphorylation regulates carbohydrate metabolism in bacteria. Microbiol Mol Biol Rev 70:939-1031

23. Kelker NE, Hanson TE, Anderson RL (1970) Alternate pathways of D-fructose metabolism in Aerobacter aerogenes - a specific D-fructokinase and its preferential role in metabolism of sucrose. J Biol Chem 245:2060-2065

24. Saier MH, Ramseier TM (1996) The catabolite repressor/activator (Cra) protein of enteric bacteria. J Bacteriol 178:3411-3417

25. Yimga MT, Leatham MP, Allen JH, Laux DC, Conway T, Cohen PS (2006) Role of gluconeogenesis and the tricarboxylic acid cycle in the virulence of Salmonella enterica serovar Tyhimurium in BALB/C mice. Infect Immun 74:1130-1140

26. Ramseier TM, Bledig S, Michotey V, Feghali R, Saier MH (1995) The global regulatory protein FruR modulates the direction of carbon flow in Escherichia coli. Mol Microbiol 16:1157-1169

27. Jung MY, Ng CY, Song H, Lee J, Oh MK (2012) Deletion of lactate dehydrogenase in Enterobacter aerogenes to enhance 2,3-butanediol production. Appl Microbiol Biotechnol 95:461-469 
28. Diancourt L, Passet V, Verhoef J, Grimont PAD, Brisse S (2005) Multilocus sequence typing of Klebsiella pneumoniae nosocomial isolates. J Clin Microbiol 43:4178-4182

29. Reid SJ, Abratt VR (2005) Sucrose utilisation in bacteria: genetic organisation and regulation. Appl Microbiol Biotechnol 67:312-321

30. Ebner R, Lengeler JW (1988) DNA-sequence of the gene scrA encoding the sucrose transport protein Enzymell ${ }^{\mathrm{scr}}$ of the phosphotransferase system from enteric bacteria-homology of the Enzymell ${ }^{\text {scr }}$ and Enzymell ${ }^{\text {bgl }}$ Proteins. Mol Microbiol 2:9-17

31. Jahreis K, Lengeler JW (1993) Molecular analysis of 2 ScrR repressors and of a ScrR-FruR hybrid repressor for sucrose and D-fructose specific regulons from enteric bacteria. Mol Microbiol 9:195-209

32. Ji XJ, Nie ZK, Huang H, Ren LJ, Peng C, Ouyang PK (2011) Elimination of carbon catabolite repression in Klebsiella oxytoca for efficient 2,3-butanediol production from glucose-xylose mixtures. Appl Microbiol Biotechnol 89:1119-1125

33. Plumbridge J (1998) Expression of ptsG, the gene for the major glucose PTS transporter in Escherichia coli, is repressed by Mlc and induced by growth on glucose. Mol Microbiol 29:1053-1063

34. Kimata K, Tanaka Y, Inada T, Aiba H (2001) Expression of the glucose transporter gene, ptsG, is regulated at the mRNA degradation step in response to glycolytic flux in Escherichia coli. EMBO J 20:3587-3595
35. Fernando S, Adhikari S, Chandrapal C, Murali N (2006) Biorefineries: current status, challenges, and future direction. Energy Fuel 20:1727-1737

36. Himmel ME, Ding SY, Johnson DK, Adney WS, Nimlos MR, Brady JW et al (2007) Biomass recalcitrance: engineering plants and enzymes for biofuels production. Science 315:804-807

37. Cherubini $F$ (2010) The biorefinery concept: using biomass instead of oil for producing energy and chemicals. Energy Convers Manag 51:1412-1421

38. Kim SR, Ha SJ, Wei N, Oh EJ, Jin YS (2012) Simultaneous co-fermentation of mixed sugars: a promising strategy for producing cellulosic ethanol. Trends Biotechnol 30:274-282

39. Shin SH, Kim S, Kim JY, Lee S, Um Y, Oh MK et al (2012) Complete genome sequence of Enterobacter aerogenes KCTC 2190. J Bacteriol 194:2373-2374

40. Datsenko KA, Wanner BL (2000) One-step inactivation of chromosomal genes in Escherichia coli K-12 using PCR products. Proc Natl Acad Sci USA 97:6640-6645

41. Jung MY, Mazumdar S, Shin SH, Yang KS, Lee J, Oh MK (2014) Improvement of 2,3-butanediol yield in Klebsiella pneumoniae by deletion of the pyruvate formate-lyase gene. Appl Environ Microbiol 80:6195-6203

\section{Submit your next manuscript to BioMed Central and take full advantage of:}

- Convenient online submission

- Thorough peer review

- No space constraints or color figure charges

- Immediate publication on acceptance

- Inclusion in PubMed, CAS, Scopus and Google Scholar

- Research which is freely available for redistribution

Submit your manuscript at

www.biomedcentral.com/submit

C Biomed Central 